\title{
Adsorption and desorption kinetics of Ga on GaN(0001): Application of Wolkenstein theory
}

\author{
Giovanni Bruno* and Maria Losurdo \\ Institute of Inorganic Methodologies and of Plasmas (IMIP)-CNR, via Orabona, 4-70126 Bari, Italy
}

Tong-Ho Kim and April Brown

Department of Electrical and Computer Engineering, Duke University, Durham, North Carolina 27708, USA

(Received 11 April 2010; revised manuscript received 9 June 2010; published 26 August 2010)

\begin{abstract}
The kinetics of $\mathrm{Ga}$ adsorption/desorption on $\mathrm{GaN}(0001)$ surfaces is investigated over the temperature range of $680-750{ }^{\circ} \mathrm{C}$ using real-time spectroscopic ellipsometry. The adsorption and desorption kinetics are described in the framework of the Wolkenstein theory, which considers not only the equilibrium between Ga adsorbed on the surface and $\mathrm{Ga}$ in the gas phase but also the electronic equilibrium at the surface. It is shown that, because of the fixed polarization charge existing at the $\mathrm{GaN}(0001)$ surface, Ga adsorption and desorption processes involve neutral and charged Ga states. By considering the GaN surface charge involved in the surface processes, we demonstrate that a second-order kinetics more accurately describes Ga desorption, in comparison with conventional models, and yields an apparent activation energy of $2.85 \pm 0.02 \mathrm{eV}$ for $\mathrm{Ga}$ desorption consistent with experiments.
\end{abstract}

DOI: 10.1103/PhysRevB.82.075326

PACS number(s): 68.43.Mn, 68.47.Fg

\section{INTRODUCTION}

The Ga adlayer present on the surface of evolving GaN (0001) films during molecular beam epitaxy (MBE) is critical to the ultimate film morphology and dopant incorporation as a result of its, impact on adatom surface diffusion and incorporation kinetics. ${ }^{1}$ Numerous studies have found that good morphology and properties GaN (0001) growth occurs under Ga-rich or near-Ga-rich conditions, suggesting that GaN surfaces are stabilized by Ga atoms. Such experimental findings have motivated research on the behavior of Ga on GaN (0001) surfaces including adsorption and desorption dynamics and the variety of surface reconstructions realized under these conditions. Various surface reconstructions evolve with increasing $\mathrm{Ga}$ on the surface. ${ }^{2}$ The pseudo- $(1 \times 1)$ structure of $\mathrm{GaN}(0001)$ is the most Ga-rich structure and is comprised of $\sim 2.3$ monolayers (MLs) (1 $\mathrm{ML}=1.14 \times 10^{15}$ atoms $\left./ \mathrm{cm}^{2}\right)$ (Ref. 3$)$ of Ga residing on top of a Ga-terminated bilayer on the surface. ${ }^{4}$ It has also been established that the unreconstructed $(1 \times 1)$ pattern corresponds to $1 \mathrm{ML}$ of $\mathrm{Ga}$ tightly bound to $\mathrm{GaN}$, with additional $\mathrm{Ga}$ adatoms on top of this layer that are responsible for surface reconstruction. ${ }^{4-6}$

The adsorption ${ }^{7}$ and desorption kinetics of Ga adlayers have been investigated in previous studies ${ }^{8,9}$ in a wide temperature range, Ga fluxes range, and in presence or absence of nitrogen during the experiment, aiming at understanding the kinetics of $\mathrm{Ga}$ adsorbate at $\mathrm{GaN}$ surfaces. A variety of techniques including mass spectrometry, ${ }^{10}$ grazing-incidence $\mathrm{X}$-ray scattering, ${ }^{11}$ and reflection high-energy electron diffraction (RHEED). 7,12,13 However, limitations exist with such techniques. For example, mass spectrometry samples $\mathrm{Ga}$ in the gas phase but not directly on the surface. Additionally, Ga coverage can only be indirectly deduced with RHEED since the Ga adlayer on the GaN surface does not diffract the electron beams directly but causes an attenuation of the RHEED beam intensity due to the disorder inherent in the layer. On the other hand, spectroscopic ellipsometry (SE) has unique advantages over other in situ monitoring tech- niques and is well suited to this particular problem. It is an optical technique and is hence noninvasive and can directly interrogate the surface and formation of overlayers with monolayer sensitivity by exploiting the real-time variation in the GaN pseudodielectric function. ${ }^{14,15}$

Indeed, an aspect that has been neglected in the previous studies is the interface charge transfer occurring during adsorption of the adsorbate layer ( $\mathrm{Ga}$ in the present case) on polar semiconductors.

We, as well as other authors, have previously reported an analysis and study of the kinetics of gallium adlayer adsorption and desorption dynamics on polar and nonpolar GaN surfaces in the "standard" framework of the Langmuir theory. ${ }^{14}$ Indeed, from a summary of the literature on the topic, it has become clear that the adsorption/desorption kinetics of $\mathrm{Ga}$ on $\mathrm{GaN}$ cannot well described by Langmuir kinetics. As already reported by Adelman et al., ${ }^{7}$ activation energy and prefactor for Ga adsorption are not constant and may vary with $\mathrm{Ga}$ surface coverage.

Indeed, it should be considered that $\mathrm{GaN}$ is a piezoelectric material with fixed polarization charge at the surface. During chemisorptions, electronic charge transfer between the GaN semiconductor and the chemisorbed species may occur, modifying the electronic structure at the surface and in the adjacent space charge region, which in return affects the chemisorption process in a nonlinear manner. This concept of electron transfer between the adsorbate and the surface has been previously considered for adsorption on $\mathrm{ZnO} .{ }^{16}$

Unlike the Langmuir's approach, Wolkenstein's theory of chemisorption takes into account these electronic interactions between the $\mathrm{GaN}$ semiconductor surface and the $\mathrm{Ga}$ adsorbate and their effect on the adsorptivity of semiconductors. ${ }^{17}$

In this work, we develop a more complete model that includes the role of electronic equilibrium at the $\mathrm{GaN}$ surface. The extension of this model in the framework of the Wolkenstein theory gives us the opportunity to further understand the complex interactions and role of the GaN evolving surface and its interaction with the Ga adlayer during gas- 
phase synthesis. Within this new framework, we examine both the adsorption and desorption kinetics of $\mathrm{Ga}$ on a $\mathrm{GaN}(0001)$ template in a regime far from $\mathrm{Ga}$-droplets formation.

In situ spectroscopic ellipsometry is used to monitor the $\mathrm{Ga}$ surface coverage in real time. For $\mathrm{Ga}$ adsorption on $\mathrm{GaN}(0001)$, the relative variation in the imaginary part of the $\mathrm{GaN}$ pseudodielectric function is directly proportional to the Ga coverage in the monolayer and submonolayer range. ${ }^{18}$ Exploiting the ability of SE to directly monitor the surface, we uncover a distinct surface phenomena related to charge transfer between the adlayer and thin film during Ga adsorption and desorption. Interpreting the kinetics of $\mathrm{Ga}$ adsorption and desorption on the $\mathrm{GaN}(0001)$ surface in the framework of the Wolkenstein theory, ${ }^{17}$ considers both chemical equilibrium between the surface and the gas phase and electronic equilibrium at the $\mathrm{GaN}$ surface. These processes involve $\mathrm{Ga}$ in two states on the $\mathrm{GaN}(0001)$ surface, specifically neutral and charged Ga. It is shown that when electronic equilibrium is considered and Ga desorption is described by a second-order kinetics, all of the desorption profiles obtained at various Ga coverages and surface temperatures result in an apparent activation energy of $2.85 \pm 0.02 \mathrm{eV}$ for desorption.

\section{EXPERIMENTAL}

Studies were performed in a VEECO Gen II MBE system equipped with RHEED and spectroscopic ellipsometry (UVISEL, Jobin Yvon) operating in the 1.5-6.5 eV photon energy range. A HVPE (hydride vapor phase epitaxy) $n$-type GaN (0001) template was used as the substrate for all of the experiments. The Ga flux was fixed at $9.63 \times 10^{-8}$ Torr, with Ga pulse times varying from 5 to $180 \mathrm{~s}$. Temperatures of 680 , 710,730 , and $750{ }^{\circ} \mathrm{C}$ were investigated. Combinations of the above parameters resulted in Ga coverage values on the GaN surface below or equal to a bilayer, ${ }^{14}$ and far from the accumulation of $\mathrm{Ga}$ droplets regime also according to the $\mathrm{Ga} / \mathrm{GaN}$ adsorption diagram reported in Ref. 8 .

The Ga adsorption/desorption kinetics was monitored recording in real time the variation in the real, $\left\langle\varepsilon_{1}\right\rangle$, and imaginary, $\left\langle\varepsilon_{2}\right\rangle$, parts of the GaN pseudodielectric function at 32 photon energies in the range $1.5-6.5 \mathrm{eV}$ with a time resolution of $1 \mathrm{~s}$ using a phase-modulated spectroscopic ellipsometer (UVISEL, Horiba Jobin-Yvon). The Ga surface coverage and/or equivalent thickness was estimated from the modeling of ellipsometric spectra knowing the dielectric function of $\mathrm{GaN}$ and $\mathrm{Ga}$ as described in Ref. 14.

\section{RESULTS AND DISCUSSION}

Figure 1 shows the variation in the imaginary part, $\left\langle\varepsilon_{2}\right\rangle$, of the GaN pseudodielectric function during $\mathrm{Ga}$ adsorption and its desorption when the Ga shutter is closed (OFF) with varying pulse time and varying temperature. The starting $\left\langle\varepsilon_{2}\right\rangle$ value is representative of the $\mathrm{GaN}$ template dielectric function; and the increase in $\left\langle\varepsilon_{2}\right\rangle$ is proportional to the Ga coverage. The adsorption of $\mathrm{Ga}$ results in a monotonic increase in $\left\langle\varepsilon_{2}\right\rangle$, indicating that the Ga surface coverage evolves
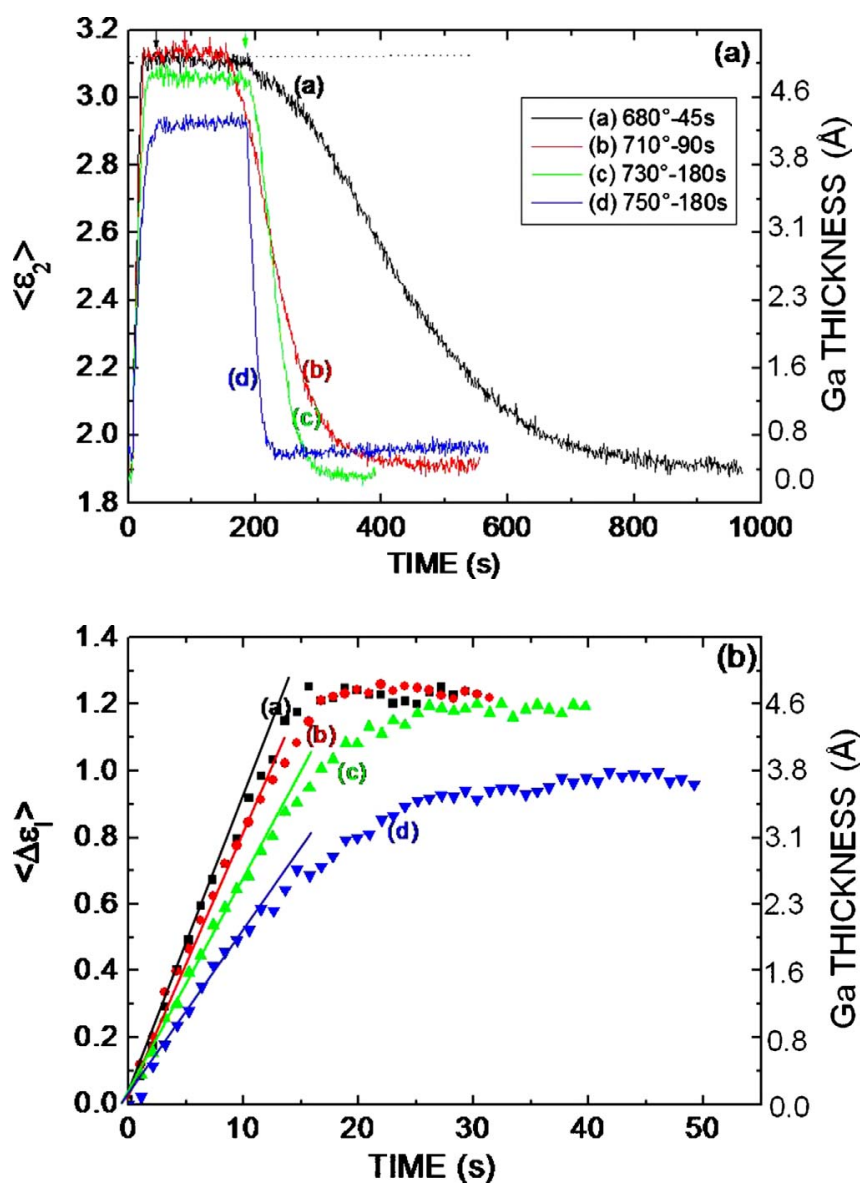

FIG. 1. (Color online) (a) Variation in the imaginary part, $\left\langle\varepsilon_{2}\right\rangle$, of the GaN pseudodielectric function during Ga adsorption and its desorption at the $\mathrm{Ga}$ shutter $\mathrm{OFF}$, at a constant $\mathrm{Ga}$ flux of $9.63 \times 10^{-8}$ Torr for temperature and Ga pulse time of (a) $680{ }^{\circ} \mathrm{C}$ and $45 \mathrm{~s}$; (b) $710^{\circ} \mathrm{C}$ and $90 \mathrm{~s}$; (c) $730{ }^{\circ} \mathrm{C}$ and $180 \mathrm{~s}$; (d) $750{ }^{\circ} \mathrm{C}$ and $180 \mathrm{~s}$. The dotted line represents the Ga bilayer level. (b) Expanded region of adsorption curves. The lines represent the fitting to obtain the neutral adsorption rate constant in Table I.

continuously ${ }^{1}$ up to a critical value, which depends on temperature and Ga flux. The ellipsometric data, or value of $\left\langle\varepsilon_{2}\right\rangle$, can be converted to an equivalent Ga thickness (in MLs) by modeling $\left\langle\varepsilon_{2}\right\rangle$ with a simple two-layer optical model consisting of the layered $\mathrm{GaN}$ substrate/Ga in air and knowing the dielectric function of $\mathrm{GaN}$ and $\mathrm{Ga}^{15}$ Using this method, we also report corresponding $\mathrm{Ga}$ coverage in the figure. It should be noted that the initial GaN substrate value is restored upon desorption of the $\mathrm{Ga}$ adlayer, indicating that the Ga desorption is complete.

Figure 2 shows the variation in $\left\langle\varepsilon_{2}\right\rangle$ during Ga adsorption with increasing Ga pulse duration and during the subsequent corresponding desorption cycle. Interestingly, different phenomena can be inferred from the variation in the shapes of the desorption curves and depend upon the Ga pulse time (i.e., the Ga surface coverage). For temperatures of 680 and $710{ }^{\circ} \mathrm{C}$, we found in earlier work using SE that the $\mathrm{Ga}$ adlayer critical thickness at the steady state is $4.8 \AA$, ${ }^{15}$ which agrees well with the thickness estimated for a laterally contracted Ga bilayer. ${ }^{2}$ The average vertical separation between the $\mathrm{GaN}$ and the first $\mathrm{Ga}$ layer has been calculated to be 


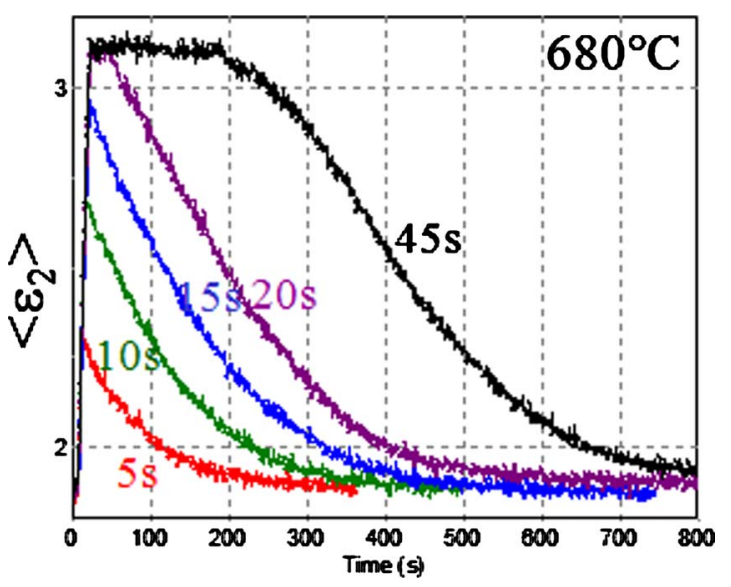

FIG. 2. (Color online) Variation in $\left\langle\varepsilon_{2}\right\rangle$ during Ga adsorption at increasing Ga pulse duration and during corresponding desorption. The Ga flux is fixed at $9.63 \times 10^{-8}$ Torr.

2.47-2.54 $\AA$, while an average vertical separation of $2.37 \AA$ has been calculated between the first and second $\mathrm{Ga}$ layers). ${ }^{2}$ As shown in Fig. 1(b), the critical Ga thickness decreases with increasing temperature: an observation that can be rationalized by a decrease in the adsorption rate constant $k_{1}$ and an increase in desorption rate constant $k_{-1}$ with the increase in the temperature (see discussion below). At higher temperatures of 730 and $750{ }^{\circ} \mathrm{C}$, the critical $\mathrm{Ga}$ coverage corresponding to the bilayer can be reached by increasing the incident $\mathrm{Ga}$ flux from $9.63 \times 10^{-8}$ to $1.86 \times 10^{-7}$ Torr, as demonstrated in our previous works, ${ }^{14}$ and consistently with the phase diagram in Ref. 8 .

Herein, we show that experimental data of Ga adsorption and desorption behavior on Ga-polar GaN can be rationalized using the Wolkenstein theory for adsorption on semiconductors in which the surface Fermi level and/or the surface charge plays a dominant role in adsorbate chemisorption. According to Wolkenstein's theory, ${ }^{17}$ localized electronic states are created into the semiconductor band gap by chemisorbed species. These states serve as traps for electrons or holes (acceptorlike or donorlike states, respectively), depending on their nature. The cardinal feature of Wolkenstein's theory is that adsorbed species, depending on electron transitions between those states and semiconductors bands, may be chemisorbed on the semiconductor surface in three ways: (1) "weak" chemisorption involving a neutral adsorbate species: in this case free carriers (electron or holes) from the substrate do not participate in the adsorption process; (2) "strong acceptor chemisorption" occurring when an electron from the surface is captured by the adsorbate species and denoted as $\mathrm{CeL}$ (where eL denotes the free electron participating from the substrate); (3) "strong donor chemisorption" occurring when an hole is captured by the adsorbate species and denoted as $\mathrm{CpL}$ ( $\mathrm{pL}$ is the free hole from the substrate).

The possibility of those different types of chemisorption stems from the ability of the chemisorbed specie to draw or donate a free electron and/or hole from/to the substrate lattice with consequent variation in surface band bending.

A diagram representing these three forms of chemisorption is shown in Fig. 3. Furthermore, one form of the chemi- (a)
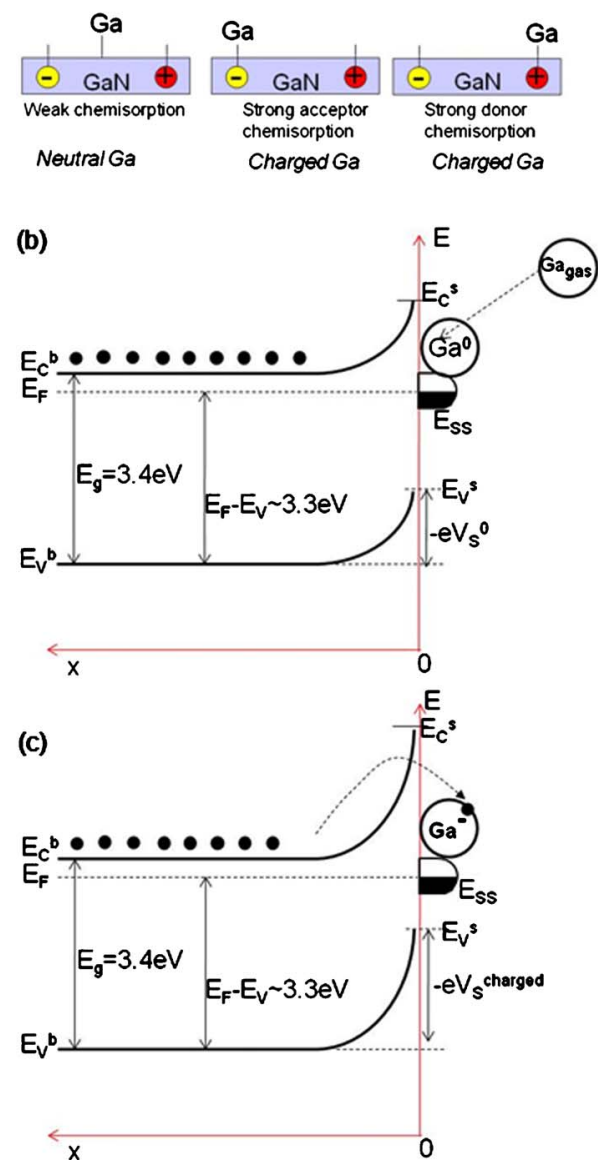

FIG. 3. (Color online) (a) Sketch of the various forms of chemisorption according to the Wolkenstein theory for the GaN/Ga. Energy-band diagram for depletive chemisorption of an acceptorlike adsorbate (gallium) on $n$-type $\mathrm{GaN}$ : (b) at the beginning of chemisorption (zero coverage); (b) after electron transfer from $\mathrm{GaN}$ to adsorbate forming charged $\mathrm{Ga}$. $\mathrm{Ga}_{\text {gas }}, \mathrm{Ga}^{0}$, and $\mathrm{Ga}^{-}$designate a free $\mathrm{Ga}$ in the gas phase, a neutral $\mathrm{Ga}$ adsorbate, and a negatively charged adsorbed $\mathrm{Ga}$, respectively. $E_{\mathrm{C}}, E_{\mathrm{V}}$, and $E_{\mathrm{F}}$ are the bulk conduction band, valence band, and the Fermi level, respectively. The superscripts "b" and "s" denote bulk and surface properties, respectively. $E_{\mathrm{ss}}$ indicate the energy level of surface charge.

sorption may change to another depending upon temperature, pressure, surface coverage and other factors, such as the presence of impurities.

The surface of Ga-polar GaN is characterized by the presence of a negative fixed surface polarization charge inducing a strong upward surface band bending. ${ }^{19}$ Figure 3 also shows the energy-band diagram for the case of depletive chemisorption of an acceptorlike univalent particle on a $n$-type $\mathrm{GaN}$ surface. At the beginning of chemisorption, the upward band bending exists. A neutral $\mathrm{Ga}$ atom from the gas phase [designed as $\mathrm{Ga}$ (gas)] approaching the surface may become chemisorbed as a neutral adsorbate (denoted as $\mathrm{Ga}^{0}$ or $\mathrm{Ga}^{*} \mathrm{~L}$ in the chemical reactions below). This is the neutral form of chemisorption, which is referred to as the weak form in Wolkenstein's theory. When chemisorbed Ga captures a free electron (denoted as - in Fig. 3 and as eL in the chemical reactions below) at the GaN surface, it becomes a chemi- 
sorbed negatively charged $\mathrm{Ga}^{-}$(denoted as $\mathrm{Ga}-\mathrm{eL}$ in the chemical reaction below). This is the charged form of chemisorption, referred to as the "strong" form in Wolkenstein's theory. Figure 3 shows that the binding energy of the charged chemisorbed $\mathrm{Ga}$ is $E_{\mathrm{B}}\left(\mathrm{Ga}^{-}\right)=\left(E_{\mathrm{C}}^{\mathrm{s}}-E_{\mathrm{ss}}\right)+e V_{\mathrm{s}}^{\text {charged }}$, where $E_{\mathrm{C}}^{\mathrm{s}}$ is the conduction band edge at the surface and $e V_{\mathrm{s}}^{\text {charged }}$ is the chemisorption-induced surface band bending, ( $e$ is the electron charge and $V_{\mathrm{s}}$ is the surface potential). Noteworthy, $e V_{\mathrm{s}}$ is a function of the coverage of chemisorbed species and therefore the adsorption heat of chemisorption of the charged form depends on its coverage. This coverage variation in the adsorption heat would be consistent with a nonconstant desorption barrier and pre-exponential factor described by Adelmann. $^{7}$

A previous independent observation of the existence of "Ga islands different states" on the GaN(0001) surface comes from Zheng et al., ${ }^{20}$ who using scanning tunneling microscopy demonstrated that there is a form of $\mathrm{Ga}$ islands that is converted to another form by voltage application, i.e., charge injection. Therefore, this work indicated that there is a change in phase of $\mathrm{Ga}$ islands involving charges.

Thus, a novel interpretation of the $\mathrm{Ga}$ adsorption/ desorption on $\mathrm{GaN}$ is presented, which consider not only the equilibrium between the chemisorbed specie $\mathrm{Ga}$ and the gas phase but also the electronic equilibrium at the surface, according to the Wolkenstein theory, discussing the three regions that can be discerned in Figs. 1 and 2: (i) the adsorption; (ii) the steady state; and (iii) the desorption. Noteworthy, the present model, based on the copresence of charged and neutral $\mathrm{Ga}$, is consistent with the bilayer formation reported from previous authors since our data indicate the 2.5 ML formation and all the analysis deals with fitting a bilayer adsorbed thickness. In fact, the presence of two different charge states of $\mathrm{Ga}$ might be argued to be a possible reason for the two different pseudomorphic layer and contracted layer formation. In fact, the various forms of chemisorption differ also in the strength of bonding between the chemisorbed particle and the lattice: when no electron or hole of the semiconductor participate in the bond, we have the so called "weak form" since capturing of an electron by an acceptor level (or of a hole by a donor level) always leads to strengthening of the chemisorptions, i.e., "charged strong form," with a continuous dynamic exchange between the charged and neutral $\mathrm{Ga}$ forms in the bilayer.

\section{A. Ga adsorption}

In studying kinetics of chemisorption on semiconductor, it is true that surface electronic equilibrium is to be achieved at the steady state of adsorption (gas phase-surface) equilibrium; however, it is not achieved at the beginning of this process. The 5 s-10 s profiles in Fig. 2 show a faster reversible monoexponential desorption as soon as the Ga shutter is closed. It has also been reported ${ }^{21}$ that only the neutral form of chemisorptions participates in the exchange with the gas phase (to maintain the electroneutrality equilibrium of the gas phase). Therefore, it can be inferred that at the initial stage of adsorption, Ga in the neutral form adsorbs "weakly" on the GaN surface. Thus, the following equilibrium between the neutral $\mathrm{Ga}$ and the gas phase applies:
TABLE I. Dependence of the adsorption constant on temperature.

\begin{tabular}{lcccc}
\hline \hline$T\left({ }^{\circ} \mathrm{C}\right)$ & 680 & 710 & 730 & 750 \\
$k_{1}\left(\mathrm{~s}^{-1}\right)$ & 0.0736 & 0.0686 & 0.0627 & 0.0584 \\
\hline \hline
\end{tabular}

$$
\mathrm{Ga}(\text { gas })+\underset{k_{-1}}{\stackrel{k_{1}}{\leftrightarrow}} \mathrm{Ga} \mathrm{L}
$$

where L denotes a lattice surface site and $\mathrm{Ga}^{*} \mathrm{~L}$ denotes adsorbed neutral $\mathrm{Ga}$.

Without electronic transitions between $\mathrm{GaN}$ and $\mathrm{Ga}$, and far from the electronic equilibrium, the ordinary Langmuir theory applies in this region of low coverage, i.e., the rate of adsorption, $r_{a d s}$, of neutral $\mathrm{Ga}, N^{0}$, can be expressed as

$$
\vec{r}_{a d s}=\frac{d N^{0}(t)}{d t}=k_{1}\left[N_{\infty}^{0}-N^{0}(t)\right]-k_{-1} N^{0}(t),
$$

where $N_{\infty}^{0}$ is the number of adsorption centers for unit area or, the same, the maximum number of atoms which can be adsorbed on the surface (also in terms of ML), $k_{1}$ is the rate constant of adsorption, and $k_{-1}$ is the rate constant of desorption of neutral $\mathrm{Ga}\left(k_{1}=1 / \tau\right.$, where $\tau$ is the lifetime of adsorbed $\mathrm{Ga}$ ). In this region of low coverage, the neutral $\mathrm{Ga}$ form predominates; phenomenologically, this can be due to the fact that the strong charged form has a higher energy, which is equal to the increased band bending and surface potential (see Fig. 3), although we cannot exclude also the existence of an additional energy barrier between the two forms. The neutral specie instantaneous concentration is given by

$$
N^{0}(t)=N_{\infty}^{0} \cdot k_{1} \cdot t
$$

This is consistent with the linear dependence of the $\left\langle\varepsilon_{2}\right\rangle$ increase (proportional to the Ga coverage) with time in Fig. 1(b). Therefore, the adsorption rate constant $k_{1}$ can be derived as a function of temperature, and data are reported in Table I, which shows that the rate constant for Ga adsorption decreases with the increase in temperature, i.e., the lower the temperature the more rapidly the surface/gas phase equilibrium is established, indicating that $\mathrm{Ga}$ adsorption on $\mathrm{GaN}(0001)$ is nonactivated. This is consistent with a weak form of adsorption, and is reasonable considering that an atomic specie is adsorbing (no energy for breaking bonds in molecules is needed).

Figure 4 shows that although reasonable fit is achieved using the Langmuir theory for neutral Ga adsorption for profile at $750{ }^{\circ} \mathrm{C}$ (which is characterized by lower coverage), the same Langmuir theory does not apply as well to profile at $680{ }^{\circ} \mathrm{C}$. Therefore, it is supposed that with the increase in surface coverage, a certain fraction of the weak Ga adsorbed passes to a "strong acceptor" chemisorption through an electron transition from the GaN surface (where excess of free electron exists), i.e., according to Wolkenstein (see Fig. 3): 

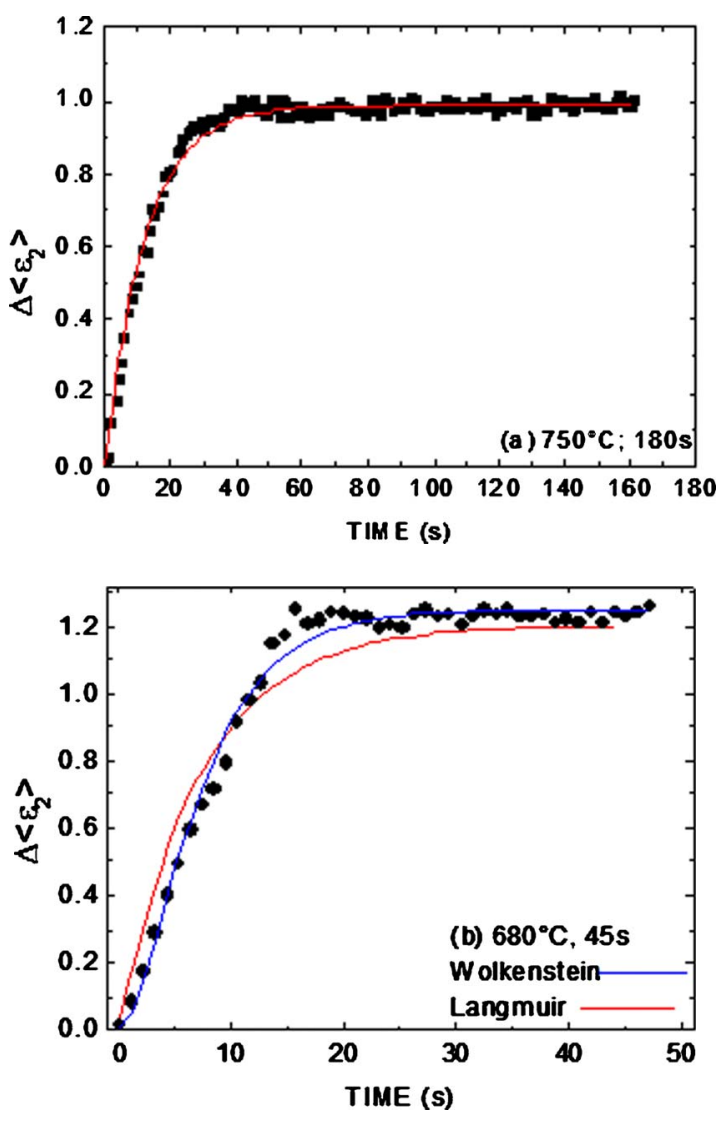

FIG. 4. (Color online) (a) and (b) fit according to the Langmuir theory for neutral $\mathrm{Ga}$ adsorption for profiles at $750{ }^{\circ} \mathrm{C}-180 \mathrm{~s}$ and $680{ }^{\circ} \mathrm{C}-45 \mathrm{~s}$, and (c) fit according to the Wolkenstein theory for charged $\mathrm{Ga}$ for profile at $680{ }^{\circ} \mathrm{C}-45 \mathrm{~s}$.

$$
\underset{\text { weak }}{\mathrm{Ga}{ }^{*} \mathrm{~L}}+\underset{k^{-} \text {strong }}{\stackrel{k^{0}}{\leftrightarrow} \mathrm{Ga}-\mathrm{eL},}
$$

where Ga-eL denotes a charged form of $\mathrm{Ga}$, and $k^{0}=1 / \tau^{0}$ denotes the probability of charging of the chemisorbed neutral $\mathrm{Ga}$ (or $\tau_{0}$ is the lifetime of the chemisorbed $\mathrm{Ga}$ in the neutral state).

At the steady state, for each temperature, adsorption equilibrium between the surface and the gas phase is established along with the electronic equilibrium at the surface with a dynamic exchange between the neutral and charged $\mathrm{Ga}$ forms, according to Eq. (4), where $k^{-}=1 / \tau^{-}$denotes the probability of neutralizing the chemisorbed charged Ga (or $\tau^{-}$is the lifetime of the chemisorbed Ga in the charged state).

Let us denote with $N^{0}$ and $N^{-}$the density of the neutral (weak) and charged (strong) chemisorbed Ga at equilibrium, being $N(t)=N^{0}(t)+N^{-}(t)$. Wolkenstein demonstrated ${ }^{17}$ that the instantaneous concentration of the neutral and charged forms of $\mathrm{Ga}$ is given by

$$
\begin{aligned}
N^{0}(t)= & \frac{N_{\infty}^{0}}{\left(\tau_{2}-\tau_{1}\right) \tau}\left\{\tau_{2}\left(\tau_{2}-\tau_{1}\right)\left[1-\exp \left(-\frac{t}{\tau_{2}}\right)\right]+\tau_{1}\left(\tau_{2}-\tau\right)\right. \\
& \left.\times\left[1-\exp \left(-\frac{t}{\tau_{1}}\right)\right]\right\},
\end{aligned}
$$

$$
N^{-}(t)=\frac{N_{\infty}}{\tau_{2}-\tau_{1}}\left\{\tau_{2}\left[1-\exp \left(-\frac{t}{\tau_{2}}\right)\right]-\tau_{1}\left[1-\exp \left(-\frac{t}{\tau_{1}}\right)\right]\right\}
$$

where $\tau_{1}$ and $\tau_{2}$ are complex functions of $\tau\left(1 / \tau=k_{-1}\right.$ is the desorption probability), $\tau^{0}$ and $\tau^{-}{ }^{22}$ These expressions for $N(t)=N^{0}(t)+N^{-}(t)$ fits well all profiles in Figs. 1, 2, and 4, validating the applicability of Wolkenstein electronic chemisorption to Ga.

\section{B. Ga desorption}

Figures 1 and 2 show that different desorption kinetics are observed depending on Ga coverage. In particular, for very low surface coverage (i.e., 5 s Ga pulse) as soon as the $\mathrm{Ga}$ shutter is closed, desorption of neutral Ga starts immediately and it follows a first-order monoexponential decay, i.e.,

$$
N(t)=N_{\infty}^{0} \exp \left(-k_{-1} \cdot t\right) .
$$

This is consistent with the presence of the neutral Ga only at low coverage, and Arrhenius analysis of desorption data as a function of temperature (for low Ga coverage) yielded an activation energy for desorption of neutral $\mathrm{Ga}$ of $2.8 \mathrm{eV}$. ${ }^{17}$ This value is well in agreement with the activation energy for desorption of $\mathrm{Ga}$ from liquid $\mathrm{Ga} .{ }^{7}$ Indeed, with the increase in $\mathrm{Ga}$ coverage (i.e., Ga pulse time), specifically when the $\mathrm{Ga}$ coverage is higher than $1 \mathrm{ML}$ (as deduced by the modeling of $\left\langle\varepsilon_{2}\right\rangle$ spectra), the decay is not monoexponential (as it can be seen from data at $20 \mathrm{~s}$ Ga pulse in Fig. 4), supporting the hypothesis that more than one $\mathrm{Ga}$ forms are present at the $\mathrm{GaN}$ surface. Moreover, when the steady-state value corresponding to the Ga bilayer thickness is reached, immediate desorption is not observed at the Ga shutter OFF differently from the short $(5 \mathrm{~s}, 10 \mathrm{~s}) \mathrm{Ga}$ pulses. A desorption delay time is observed, resulting in S-shape desorption profile (see Figs. 1 and 2). These data indicate that desorption is coverage dependent contrarily to the simplifying assumption that adsorption and desorption were independent of coverage made by Brandt et al. ${ }^{3}$

We derived $\tau=83 \mathrm{~s} \gg \tau^{0}=1.96 \mathrm{~s} \gg \tau^{-}=0.18 \mathrm{~s}$, which satisfy the Wolkenstein condition $\tau \gg \tau^{0}, \tau^{-}$; therefore, the electronic equilibrium during desorption is maintained and desorption is complete (see Fig. 1). In particular, the electronic equilibrium is maintained through two balancing processes: desorption of neutral $\mathrm{Ga}$ (weak adsorption), which violates the equilibrium, and the discharging of charged $\mathrm{Ga}$, which restores the equilibrium. This desorption mechanism can be summarized as

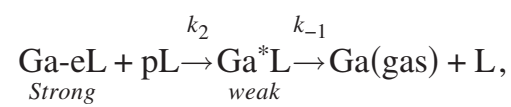

where $k_{2}$ and $k_{-1}$ are the rate constants of the discharge and desorption processes, respectively, and $\mathrm{pL}$ denotes a hole at GaN surface.

Thus,

$$
r_{\text {des }}^{\leftarrow}=-\frac{d N(t)}{d t}=k_{-1} \cdot N^{0}(t)
$$

according to reaction (7), we can write 


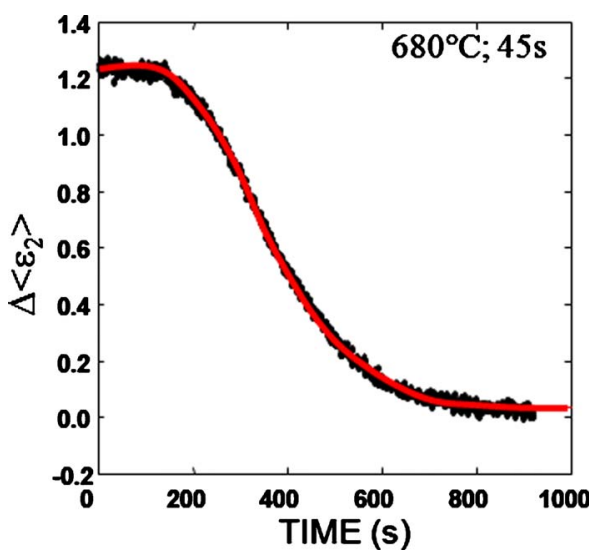

FIG. 5. (Color online) Example of the fit goodness of the $680{ }^{\circ} \mathrm{C}-45 \mathrm{~s}$ desorption profile according to Eq. (9) (see text) from Wolkenstein theory.

$$
\frac{d N^{0}(t)}{d t}=k_{2} \cdot N^{-}(t) \cdot\langle\mathrm{pL}\rangle(t)-k_{-1} \cdot N^{0}(t),
$$

where $\langle\mathrm{pL}\rangle$ indicates the surface charge density involved in the strong chemisorption and electronic equilibrium $[\langle\mathrm{pL}\rangle(t)$ indicates its time dependence). According to Eqs. (7)-(9), the rate of desorption depends on the availability of free holes at GaN surface.

Therefore, by applying the steady state to $N^{0}(t)$, the following rate equation for Ga desorption can be derived:

$$
r_{\text {des }}^{\leftarrow}=-\frac{d N(t)}{d t}=k_{2} \cdot N^{-}(t) \cdot\langle\mathrm{pL}\rangle(t)
$$

which is a second-order kinetic equation. Integration of Eq. (10) with the boundary conditions that for desorption at $t=0$ (when Ga shutter is closed), $N_{t=0}=N^{*}$, (i.e., the value determined by ellipsometric monitoring at the steady state), and $\langle\mathrm{pL}\rangle_{t=0}=\langle\mathrm{pL}\rangle^{*}$, results in the following expression for the Ga desorption:

$$
N(t)=N^{*}-\frac{N^{*} \cdot\langle\mathrm{pL}\rangle^{*} \cdot\left\{1-\exp \left[\left(\langle\mathrm{pL}\rangle^{*}-N^{*}\right) \cdot k_{2} \cdot i\right\}\right.}{\langle\mathrm{pL}\rangle^{*}+N^{*} \exp \left[\left(\langle\mathrm{pL}\rangle^{*}-N^{*}\right) \cdot k_{2} \cdot t\right]} .
$$

Figure 5 shows examples of the fit goodness of desorption profiles according to Eq. (11).

The second-order rate constant values derived for desorption of $\mathrm{Ga}$ according to process (7) are shown in the Arrhenius plot of Fig. 6, which yields an apparent activation energy $E_{\mathrm{a}}=2.85 \pm 0.02 \mathrm{eV}$ for Ga desorption, in agreement with the value for desorption from liquid $\mathrm{Ga}$, and supporting that only neutral $\mathrm{Ga}$ is desorbing from the $\mathrm{GaN}(0001)$ surface.

This model is consistent with Adelman et al., ${ }^{7}$ who introduced a temperature-dependent activation energy. Considering that the surface coverage changes by changing the temperature, the Adelman work represented advancement to modeling chemisorption with a nonconstant binding energy

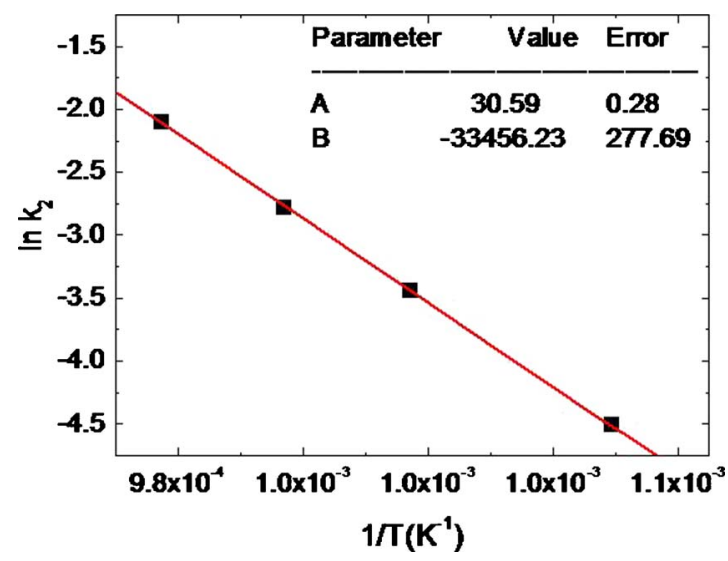

FIG. 6. (Color online) Arrhenius plot of the second-order rate constant values derived for desorption of $\mathrm{Ga}$ according to process (6) from which activation energy is derived. $\ln k_{2}=A-B / T(\mathrm{~K})$.

between the adsorbate and adsorbent. In fact, in the case of chemisorption on semiconductors, where charge transfer is involved, the binding energy (adsorption heat) varies with the degree of coverage of chemisorbed species due to the strong electronic interaction between the adsorbate and adsorbent. $^{23}$

Thus, the following analysis shows that when surface electronic equilibrium is considered in the adsorption/ desorption process of $\mathrm{Ga}$ a second-order kinetic analysis results in a unique value of apparent activation energy for $\mathrm{Ga}$ desorption independent of surface coverage. Conversely, in the simplifying assumption of first-order desorption widely applied in the previous literature resulted in a wide range of values for the activation energy.

\section{CONCLUSIONS}

In summary, the Ga adsorption and desorption kinetics on $\mathrm{GaN}(0001)$ surface have been directly monitored in real time using spectroscopic ellipsometry in the temperature range $680-750{ }^{\circ} \mathrm{C}$. Results indicate that charge transfer between the Ga-polar $\mathrm{GaN}$ and the Ga adsorbate occurs stabilizing the bilayer at the $\mathrm{GaN}(0001)$ surface. The Ga adsorption and desorption kinetics have been modeled in the frame of the Wolkenstein theory of chemisorption on semiconductors, which also consider electronic equilibrium at the surface. In the frame of this theory, neutral and charged Ga states are involved in the adsorption on Ga-polar GaN. This can be understood considering that for ionic polar surface to be stabilized, the surface charge density must be modified so as to balance the polarization electric field, and the charge compensation is operated by the Ga surface metallization.

\section{ACKNOWLEDGMENT}

The authors acknowledge Soojong Choi for her contribution to discussion and collection of data. 
*Corresponding author. FAX: +39-0805443562; giovanni.bruno@ba.imip.cnr.it

${ }^{1}$ G. Koblmüller, J. Brown, R. Averbeck, H. Riechert, P. Pongratz, and J. S. Speck, Jpn. J. Appl. Phys. 44, L906 (2005).

${ }^{2}$ J. E. Northrup, J. Neugebauer, R. M. Feenstra, and A. R. Smith, Phys. Rev. B 61, 9932 (2000).

${ }^{3}$ O. Brandt, Y. J. Sun, L. Daweritz, and K. H. Ploog, Phys. Rev. B 69, 165326 (2004).

${ }^{4}$ A. R. Smith, R. M. Feenstra, D. W. Greve, M. S. Shin, M. Skowronski, J. Neugebauer, and J. E. Northrup, J. Vac. Sci. Technol. B 16, 2242 (1998).

${ }^{5}$ T. Zywietz, J. Neugebauer, and M. Scheffler, Appl. Phys. Lett. 73, 487 (1998)

${ }^{6}$ J. Neugebauer, T. Zywietz, M. Scheffler, J. E. Northrup, and C. G. Van de Walle, Phys. Rev. Lett. 80, 3097 (1998).

${ }^{7}$ C. Adelmann, J. Brault, G. Mula, B. Daudin, L. Lymperakis, and J. Neugebauer, Phys. Rev. B 67, 165419 (2003).

${ }^{8}$ J. S. Brown, G. Koblmüller, F. Wu, R. Averbeck, H. Riechert, and J. S. Speck, J. Appl. Phys. 99, 074902 (2006).

${ }^{9}$ L. He, Y. T. Moon, J. Xie, M. Munoz, D. Johnstone, and H. Morkoc, Appl. Phys. Lett. 88, 071901 (2006).

${ }^{10}$ G. Koblmüller, R. Averbeck, H. Riechert, and P. Pongratz, Phys. Rev. B 69, 035325 (2004).

${ }^{11}$ A. S. Özcan, Y. Wang, G. Ozaydin, and K. F. Ludwig, J. Appl. Phys. 100, 084307 (2006).

${ }^{12}$ C. Adelmann, J. Brault, D. Jalabert, P. Gentile, H. Mariette, G.
Mula, and B. Daudin, J. Appl. Phys. 91, 9638 (2002).

${ }^{13}$ B. Heying, R. Averbeck, L. F. Chen, E. Haus, H. Riechert, and J. S. Speck, J. Appl. Phys. 88, 1855 (2000).

${ }^{14}$ S. Choi, T.-Ho Kim, H. O. Everitt, A. Brown, M. Losurdo, G. Bruno, and A. Moto, J. Vac. Sci. Technol. B 25, 969 (2007).

${ }^{15}$ S. Choi, T. H. Kim, H. O. Everitt, A. Brown, M. Losurdo, G. Bruno, and A. Moto, Appl. Phys. Lett. 89, 181915 (2006).

${ }^{16}$ W. Göpel and G. Rocker, J. Vac. Sci. Technol. 21, 389 (1982).

${ }^{17}$ T. Wolkenstein, Electronic Processes on Semiconductor Surfaces during Chemisorption (Consultants Bureau, New York, 1991), Chap. 3, pp. 83-124.

${ }^{18}$ S. Andrieu and F. A. d'Avitaya, J. Cryst. Growth 112, 146 (1991).

${ }^{19}$ S. Sabuktagin, M. A. Reshchikov, D. K. Johnstone, and H. Morkoc, GaN, AlN, InN, and Their Alloys, MRS Symposia Proceedings No. 831 (Materials Research Society, Pittsburgh, 2004), p. Y5.39.

${ }^{20}$ L. X. Zheng, M. H. Xie, S. M. Seutter, S. H. Cheung, and S. Y. Tong, Phys. Rev. Lett. 85, 2352 (2000).

${ }^{21}$ T. H. Wolkenstein, The Electron Theory of Catalysis on Semiconductors (Pergamon Press, Oxford, 1963); O. Peshev, J. Res. Inst. Catalysis, Hokkaido Univ. 16, 217 (1968).

${ }^{22}$ From Ref. 15, p. 102, $\frac{1}{\tau_{1}}=\lambda\left(1+\sqrt{1-\frac{\mu}{\lambda^{2}}}\right), \frac{1}{\tau_{2}}=\lambda\left(1-\sqrt{1-\frac{\mu}{\lambda^{2}}}\right) ; \lambda$ $=\frac{1}{2}\left(\frac{1}{\tau}+\frac{1}{\tau^{0}}+\frac{1}{\tau^{-}}\right), \mu=\frac{1}{\tau} \frac{1}{\tau^{-}}$.

${ }^{23}$ T. Engler and W. Lorenz, Surf. Sci. 104, 549 (1981). 